\title{
Perfil clínico, sociodemográfico e funcional de indivíduos com aneurisma cerebral internados em um hospital de referência em Salvador/BA
}

\author{
Clinical, sociodemographic and functional profile \\ of individuals with cerebral aneurysm interned in a \\ reference hospital in Salvador, Bahia, Brazil
}

\section{Vanessa Cristina Santos de Oliveira ${ }^{1}$ Ariana Prazeres dos Santos ${ }^{2}$ (1) Jorge Luis Motta dos Anjos ${ }^{3}$ (1) Isabella Pereira Rosa de Castro ${ }^{4}$ (1)}

\author{
1Secretaria da Saúde do Estado da Bahia (Salvador). Bahia, Brasil. vancrisfisio@gmail.com \\ ${ }^{2}$ Autora para correspondência. Secretaria da Saúde do Estado da Bahia (Salvador). Bahia, Brasil. ary_prazeres12@hotmail.com \\ 3,4 Hospital Roberto Santos (Salvador). Bahia, Brasil. jorgelmanjos@hotmail.com, bellaprosa@hotmail.com
}

\begin{abstract}
RESUMO | INTRODUÇÃo: Aneurisma cerebral (AC) é a dilatação anormal de uma artéria que irriga o cérebro. Cerca de 3,2\% da população mundial apresenta algum AC com potencial risco de evoluir para hemorragia subaracnóidea (HSA). OBJETIVO: Descrever o perfil clínico, sociodemográfico e funcional de indivíduos com diagnóstico clínico de AC atendidos em Salvador/BA. MÉTODO: Estudo observacional e transversal, realizado em um hospital de referência em Salvador/BA. Indivíduos maiores de 18 anos com diagnóstico de $A C(N=25)$ roto ou não roto, foram avaliados o perfil sociodemográfico (idade, sexo, escolaridade e ocupação), clinico (comorbidades, hábitos de vida e nível de consciência através da ECG, Escala de Coma de Glasgow) e funcional (MRC, Medical Research Council; ECT, Escala de Comprometimento de Tronco e Rankin Modificada). Variáveis qualitativas foram apresentadas por frequências relativas e as variáveis quantitativas por meio de médias, desvio-padrão ou medianas e intervalo mínimo e máximo. RESULTADOS: Foram incluídos 13 indivíduos diagnosticados com AC, idade mediana de 51 , sendo $84,6 \%$ do sexo feminino, $30,8 \%$ ativos no mercado de trabalho. $61,5 \%$ da população apresentou AC não roto e ECG 15. Observamos HAS em $53,8 \%$ e obesidade em $7,7 \%$. Cerca de $61,5 \%$ eram sedentários, $15,4 \%$ etilistas e $30,8 \%$ tabagistas. Na avaliação da funcionalidade, o escore MRC mediano foi de 54, Rankin a mediana foi 4 e na ECT de 19,5. CONCLUSÃo: Os achados demonstram uma predominância dos AC em mulheres e declínio funcional dos indivíduos durante o internamento, tornando-se necessário intervenções que visem minimizar possíveis perdas funcionais nesta população.
\end{abstract}

PALAVRAS-CHAVE: Aneurisma intracraniano. Classificação Internacional de Funcionalidade. Incapacidade e Saúde. Perfil de Saúde.
ABSTRACT | INTRODUCTION: Cerebral aneurysm (AC) is the abnormal dilation of an artery that irrigates the brain. About $3.2 \%$ of the world population has some CA with a potential risk of developing subarachnoid hemorrhage (SAH). OBJECTIVE: To describe the clinical, sociodemographic and functional profile of individuals with a clinical diagnosis of CA treated in Salvador / BA. METHODS: Observational and cross-sectional study, carried out in a reference hospital in Salvador / BA. Individuals over 18 years of age diagnosed with $C A(N=25)$ ruptured or not, the sociodemographic profile (age, sex, education and occupation), clinical (comorbidities, lifestyle and level of consciousness through ECG, Scale Glasgow Coma) and functional (MRC, Medical Research Council; ECT, Trunk Compromise Scale and Modified Rankin). Qualitative variables were presented by relative frequencies and quantitative variables by means of means, standard deviation or medians and minimum and maximum intervals. RESULTS: 13 individuals diagnosed with CA were included, with a median age of $51,84.6 \%$ of whom were female, $30.8 \%$ were active in the labor market. $61.5 \%$ of the population had a diagnosis of non-ruptured AC and ECG 15. We observed SAH in 53.8\% and obesity in $7.7 \%$. About $61.5 \%$ were sedentary, $15.4 \%$ drinkers and $30.8 \%$ smokers. In the evaluation of functionality, the median MRC score was 54 , Rankin the median was 4 and in ECT it was 19.5. CONCLUSION: The findings demonstrate a predominance of CA in women and functional decline of individuals during hospitalization, making interventions necessary to minimize possible functional losses in this population.

KEYWORDS: Intracranial aneurysm. International Classification of Functionality. Disability and Health. Health Profile. 


\section{Introdução}

O aneurisma cerebral (AC) é uma condição patológica descrita como uma alteração anatômica na parede dos vasos atingindo a circulação sanguínea. Cerca de $3,2 \%$ da população mundial apresenta algum tipo de aneurisma cerebral com potencial risco de evoluir para hemorragia subaracnóidea (HSA) ou acidente vascular cerebral hemorrágico (AVCH) ${ }^{1}$. No Brasil a ruptura de aneurismas e a HSA são as causas mais frequentes de óbitos na população adulta com grande potencial para morbimortalidade e hospitalização, sendo considerado como um problema de saúde pública ${ }^{1,2}$. O tratamento em centros especializados é o maior responsável pela diminuição dessas taxas ${ }^{3}$.

Considerado como um evento multifatorial, o surgimento de aneurismas intracranianos conta com componentes familiares, intrínsecos e extrínsecos ao indivíduo. As evidências apontam que pessoas que apresentam história familiar de AC possuem um risco acentuado para desenvolvê-los, quando comparado com a população em geral. Os demais fatores de risco apontam a idade, sexo feminino, hipertensão arterial sistêmica (HAS), tabagismo, etilismo, dislipidemias e diabetes mellitus (DM) como potenciais agravadores para o surgimento de $\mathrm{AC}^{4,5}$.

De acordo com Tiensoli et al., a caracterização da população atendida por determinado serviço é uma forma disponível para se estabelecer prioridades e poder realizar o planejamento estratégico que produza resultados satisfatórios, sendo considerado imprescindível. Já é sabido que indivíduos com AC podem apresentar alterações neurológicas significativas que podem causar importantes disfunções e incapacidades funcionais ${ }^{7}$. Porém, são escassos estudos que analisaram o perfil funcional de indivíduos com AC em uma unidade de internamento hospitalar. Nesse contexto, o objetivo do estudo foi descrever o perfil clínico, sociodemográfico e funcional de indivíduos internados em um hospital público de grande porte, com diagnóstico clínico de AC.

\section{Materiais e métodos}

Trata-se de um estudo transversal de caráter descritivo, com amostragem por conveniência consecutiva, em que os participantes foram recrutados em uma unidade de internamento de um Hospital de Referência em Salvador- BA. Os dados foram coletados de uma base de dados de um estudo maior, intitulado "Avaliação funcional de indivíduos após lesão neurológica em um hospital de grande porte em Salvador- BA" e foi realizado no período de Agosto de 2017 a Junho de 2018.

Os critérios de inclusão foram apresentar diagnóstico de $A C$ roto ou não roto (por um neurocirurgião), comprovados por exame de imagem (Tomografia Computadorizada e/ou Ressonância Magnética de crânio), e maiores de 18 anos. Os critérios de exclusão foram outra condição neurológica que não a $A C$, associação a lesão expansiva intracerebral e/ou a AVCH ou que tivessem realizado cirurgia para reparo do aneurisma.

O estudo foi aprovado pelo Comitê de Ética e Pesquisa (CAAE 59587316.1.0000.5028) e todos participantes assinaram o Termo de Consentimento Livre e Esclarecido (TCLE) concordando com sua participação na pesquisa.

Para analisar os dados sociodemográficos foi elaborada pelos autores uma entrevista contendo informações necessárias para traçar o perfil dos pacientes como: idade, gênero, escolaridade, ocupação e comorbidades (hipertensão arterial, diabetes melittus, insuficiência renal, obesidade e cardiopatias).

A Escala de Coma de Glasgow (ECG) foi utilizada para testar o nível de consciência. A ECG é um teste válido, confiável e sensível comumente utilizado após lesão cerebral. As sessões avaliadas são: abertura ocular (nenhuma, ao estímulo doloroso, ao estímulo verbal, espontânea), resposta motora (nenhuma, extensão, flexão anormal, retirada, localização da dor, obediência aos comandos), resposta verbal (nenhuma, incompreensível, imprópria, confusa, orientada) ${ }^{8}$. 
O perfil funcional do paciente foi traçado a partir da coleta de dados importantes para mensurar o nível de mobilidade e a funcionalidade do paciente. Utilizamos o escore Medical Counsil Research (MRC) para avaliar a força muscular dos indivíduos, separada em 12 grupos musculares, um escore total abaixo de 48/60 designa fraqueza significativa, e escore total MRC abaixo de 36/48 indica fraqueza grave?

A escala de Rankin modificada foi utilizada para quantificar o grau de incapacidade e dependência funcional dos indivíduos ao realizarem atividades de vida diária. Ela é dividida em seis graus, onde o grau zero corresponde aos indivíduos sem sintomas residuais ou incapacidade e o grau cinco aos indivíduos com incapacidade grave, restrito ao leito ou à cadeira e o grau seis, que corresponde à morte ${ }^{10}$.

A presença de déficit de controle de tronco foi mensurada através da Escala de Comprometimento de Tronco (ECT). Esta é composta por sete itens que avaliam a verticalidade e força abdominal do indivíduo. A pontuação de cada item varia de 0-3, sendo 21 pontos a pontuação máxima. Quanto maior a pontuação, menor o comprometimento do controle de tronco ${ }^{11}$.

A análise estatística dos dados foi processada pelo software SPSS (Statistical Package for Social Sciences) para Windows, versão 16.0. Foi feita a análise descritiva das variáveis estudadas, onde as variáveis qualitativas foram apresentadas por meio de frequências relativas (percentuais) e as variáveis quantitativas/numéricas por meio de médias, desvio-padrão ou medianas e intervalo mínimo e máximo.

\section{Resultados e discussão}

A amostra inicial contou com 25 indivíduos potencialmente elegíveis, sendo examinados de acordo com critérios de inclusão, onde 12 indivíduos foram excluídos por haverem realizado cirurgia de reparo do AC, o que poderia gerar viés nos resultados. A amostra final contempla dados de 13 indivíduos elegíveis de fato para este estudo. A pesquisa apresenta limitações quanto ao número da amostra e dados perdidos em algumas variáveis investigadas. A idade mediana entre a população estudada foi de 51 (37,25 - 61,25), sendo a mínima de 25 anos e a máxima de 74 anos. Quanto a prevalência entre gêneros, 11 eram do sexo feminino correspondendo a $84,6 \%$ da amostra. Somente 4 indivíduos (30,8\%) encontravam- se ativos no mercado de trabalho, dado não conclusivo visto que houveram perdas. A média de escolaridade em anos foi de $8(4,5000-9,5000)$, o que sugere que alguns dos indivíduos não concluíram o ensino médio, dados descritos na tabela 1.

Tabela 1. Dados Sociodemográficos

\begin{tabular}{|c|c|c|}
\hline Variáveis & & $\mathrm{N}: 13$ \\
\hline $\begin{array}{l}\text { Idade Mediana (IQ) * } \\
\text { Idade Mínima } \\
\text { Idade Máxima }\end{array}$ & & $\begin{array}{l}51,00(37,25-61,25) \\
25 \\
74\end{array}$ \\
\hline \multicolumn{3}{|l|}{ Sexo n (\%) } \\
\hline & $\begin{array}{l}\text { Masculino } \\
\text { Feminino }\end{array}$ & $\begin{array}{l}2(15,4) \\
11(84,6)\end{array}$ \\
\hline $\begin{array}{l}\text { Escolaridade Mediana }(I Q)^{*} \\
\text { Escolaridade Mínimo } \\
\text { Escolaridade Máximo }\end{array}$ & & $\begin{array}{l}8,0000(4,5000-9,5000) \\
3,00 \\
12,00\end{array}$ \\
\hline Ocupação n (\%)* & $\begin{array}{l}\text { Aposentado } \\
\text { Em atividade } \\
\text { Desempregado }\end{array}$ & $\begin{array}{l}2(15,4) \\
4(30,8) \\
3(23,1)\end{array}$ \\
\hline
\end{tabular}

$\overline{I Q}$, intervalo Interquartil. Fonte: Elaborado pelos autores.

*Percentuais não totais em função de dados perdidos. 
Analisando a prevalência entre gêneros, Isaías et al. .12 avaliaram 51 pacientes da rede pública hospitalar de Teresina, havendo predominância do gênero feminino com um percentual de $74,51 \%$ dos participantes totais da pesquisa, onde indivíduos com 51 e 60 anos de idade foram mais susceptíveis a desenvolver um AC, representando $35,30 \%$ da amostra. Steinkirch et al. $\frac{13}{2}$ avaliaram dados sociodemográficos de indivíduos tratados no Instituto de Neurologia de Curitiba (INC) com um número total de 254 pacientes, destes $73,7 \%$ eram mulheres, com idades mais prevalentes entre 50 e 59 anos, corroborando com os resultados atuais desta pesquisa.

Em contrapartida, Faleiro et al. ${ }^{14}$ publicaram um estudo com 824 indivíduos com diagnóstico clínico de AC internados no serviço de neurocirurgia em Belo Horizonte, onde a prevalência foi de $59 \%$ do gênero masculino e a faixa etária entre 41 e 60 anos de idade. Um estudo ${ }^{15}$ sugere que pessoas do gênero feminino possuem algum fator predisponente ao desenvolvimento de aneurismas, devido a sua maior prevalência no gênero supracitado.

A maior parte da população estudada apresentou diagnóstico de aneurisma não roto $N=8(61,5 \%)$ e nível de consciência preservado, segundo avaliação da ECG com pontuação ${ }^{15}$, no entanto houveram perdas dessa variável com potencial risco de viés. Dentre as comorbidades investigadas, as mais prevalentes foram a HAS N=7 (53,8\%) seguida pela obesidade $\mathrm{N}=1(7,7 \%)$. Com relação aos hábitos de vida, $\mathrm{N}=8(61,5 \%)$ eram sedentários, $\mathrm{N}=2(15,4 \%)$ etilistas e $\mathrm{N}=4(30,8 \%)$ tabagistas, como exposto na tabela 2.

Tabela 2. Características Clínicas

\begin{tabular}{|c|c|c|c|}
\hline Variáveis & & & $\mathrm{N}: 13$ \\
\hline \multicolumn{4}{|l|}{ Diagnóstico n (\%) } \\
\hline & Aneurisma não roto & & $8(61,5)$ \\
\hline & Aneurisma roto & & $5(38,5)$ \\
\hline \multicolumn{4}{|l|}{ Comorbidades n (\%) } \\
\hline & HAS & & \\
\hline & & Sim & $7(53,8)$ \\
\hline & & Não & $6(46,2)$ \\
\hline & DM & & \\
\hline & & Sim & - \\
\hline & & Não & $13(100)$ \\
\hline & IRPA & & \\
\hline & & Sim & - \\
\hline & & Não & $13(100)$ \\
\hline & Obesidade & & \\
\hline & & Sim & $1(7,7)$ \\
\hline & & Não & $12(92,3)$ \\
\hline & Cardiopatia & & \\
\hline & & Sim & - \\
\hline & & Não & $13(100)$ \\
\hline \multicolumn{4}{|l|}{ Hábitos de vida n (\%) } \\
\hline & Etilismo & & \\
\hline & & Sim & $2(15,4)$ \\
\hline & & Não & $11(84,6)$ \\
\hline & Tabagismo & & \\
\hline & & Sim & $4(30,8)$ \\
\hline & & Não & $9(69,2)$ \\
\hline & Sedentarismo & & \\
\hline & & Sim & $8(61,5)$ \\
\hline & & Não & $5(38,5)$ \\
\hline Glasgow Mediana (IQ)* & & & $15,00(15,00-15,00)$ \\
\hline Glasgow Mínimo & & & 15,00 \\
\hline Glasgow Máximo & & & 15,00 \\
\hline
\end{tabular}

HAS, Hipertensão arterial sistêmica; DM, Diabetes Mellitus; IRPA, Insuficiência Respiratória Aguda; Fonte: Elaborado pelos autores 
Como observado na tabela 2 há uma prevalência de aneurismas não rotos (61,5\%), corroborando com Steinkirch ${ }^{13}$ onde cerca de 75,9\% da amostra, eram indivíduos com aneurismas não rotos. Do número total da amostra 53,8\% dos indivíduos tinham HAS prévia, reforçando dados de um estudo $\frac{15}{15}$ onde a HAS foi citada em $38,21 \%$ dos casos e a DM em 5,69\%, não sendo citadas outras comorbidades associadas ao AC. Em contrapartida um estudo demonstrou associação do AC a HAS (44\%), tabagismo (12,4\%) e a dislipidemia em (11,3\%). Estes achados confirmam uma hipótese em investigação associada a etiologia dos aneurismas, onde a causa inicial para a formação de um aneurisma ainda é incerta, mas existem evidências ${ }^{13}$ de que fatores genéticos e hemodinâmicos estejam envolvidos. Secundariamente, podem ser desenvolvidos por aterosclerose, vasculopatias e malformações arteriovenosas (MAV), associando-se a fatores de risco como HAS, tabagismo, e DM no desenvolvimento e ruptura do $\mathrm{AC}^{13}$.

Em relação ao nível de consciência, dados da literatura confirmam que grande parte dos indivíduos são admitidos sem alterações significantes pontuadas pela ECG, como num estudo $\frac{12}{2}$ onde $90,2 \%$ dos indivíduos apresentaram pontuação máxima na ECG. No entanto, a ECG inicial não prediz a evolução clínica, devido a instabilidade do quadro e possível ruptura aos mínimos esforços, a depender do tamanho e localização do AC.

$\mathrm{Na}$ avaliação quantitativa da funcionalidade, a mediana do Medical Research Council (MRC) foi 54 (41 - 60) indicando que a maioria dos indivíduos tinham a força muscular preservada no momento da avaliação. Na Escala de Rankin Modificada (ERM), a mediana encontrada foi 4,00 (1,00 - 5,00), sugerindo que grande parte dos indivíduos apresentavam uma incapacidade moderada durante o internamento. A avaliação do tronco realizada pela ECT , obteve a mediana de 19,50 (0,00 - 21,00), no entanto houveram perdas desses dados, havendo risco de viés.

Tabela 3. Perfil funcional

\begin{tabular}{|c|c|c|}
\hline \multicolumn{2}{|l|}{ Variáveis } & $\mathrm{N}: 13$ \\
\hline \multirow{2}{*}{\multicolumn{2}{|c|}{$\begin{array}{l}\text { MRC Mediana (IQ)* } \\
\text { MRC Mínimo }\end{array}$}} & $54,00(41,00-60,00)$ \\
\hline & & 13 \\
\hline \multirow{2}{*}{\multicolumn{2}{|c|}{ MRC Máximo }} & 60 \\
\hline & & MRC n (\%)* \\
\hline & 13 & $1(7,7)$ \\
\hline & 38 & $1(7,7)$ \\
\hline & 50 & $1(7,7)$ \\
\hline & 54 & $2(15,4)$ \\
\hline & 60 & $3(23,1)$ \\
\hline
\end{tabular}

\section{RANKIN Mediana (IQ) \\ RANKIN Mínimo \\ RANKIN Máximo \\ RANKIN n (\%)}

$\begin{array}{ll} & 4,00(1,00-5,00) \\ & 0 \\ & 5 \\ & \\ \text { Assintomático } & 2(15,4) \\ \text { Sintomático s/ incapacidade } & 4(30,8) \\ \text { Incapacidade Moderada a grave } & 1(7,7) \\ \text { Incapacidade Grave } & 6(46,2) \\ & \\ & 19,50(0,00-21,00) \\ & 0 \\ 16 & 21 \\ 18 & 1 \\ 21 & 1 \\ & 5\end{array}$

ECT Mediana (IQ)*

ECT Mínimo

ECT Máximo

ECT $n(\%)$ *

MRC, Medical Research Council; ECT, Escala de Comprometimento de Tronco. Fonte: Elaborado pelos autores

*Percentuais não totais em função de dados perdidos. 
Existem poucos trabalhos que relacionam o perfil e evolução funcional de pacientes com AC. É sabido que a principal consequência da ruptura de um aneurisma, é a hemorragia subaracnóide (HSA) que pode ser quantificada de acordo a gravidade pela escala de fisher e os achados tomográficos pela escala de Hunt-Hess.

Mocco et al. $\frac{16}{}$ denominam os déficits motores associados ao AC em hemiplegia e hemiparesia, secundários a ruptura ou vasoespasmos. Os déficits motores exatos dependem da localização do aneurisma, da hemorragia subsequente e da intensidade do sangramento. Um estudo ${ }^{17}$ encontrou déficits motores presentes em $31,1 \%$ dos indivíduos internados por AC corroborando com outro estudo $\frac{12}{12}$ onde foi observado aumento no número de não deambuladores no momento da alta, expressos pela pontuação de marcha ausente em $15,69 \%$ indivíduos no momento da admissão e $27,45 \%$ no momento da alta hospitalar.

Em contrapartida, um estudo $\frac{18}{}$ com 13 indivíduos avaliou dados da Medida de Independência Funcional (MIF) nos domínios cognitivos e motor, sendo observado melhora desses domínios durante o internamento, expressos por pontuação média de 33,23 na admissão e 60.85 pontos na alta. A justificativa para a melhora dos déficits motores, é o desfecho no pósoperatório de clipagem ou embolização do $\mathrm{AC}^{12}$.

\section{Considerações finais}

De acordo com os dados aqui contidos, observamos uma predominância dos aneurismas cerebrais em mulheres, contendo variáveis que precisam ser meIhor estudadas para determinar as possíveis causas deste achado. Outro ponto importante é frisar o declínio funcional dos pacientes no ambiente intra-hospitalar em indivíduos com AC, o que pode ser explicado por ruptura aneurismática, vasoespasmo, ou imobilidade. Este declínio funcional pode ser progressivo e/ ou perdurar ao longo de meses e anos em casos de indivíduos com HSA, estudos longitudinais são necessários para investigar este fato em indivíduos com AC não-rotos, e possíveis medidas fisioterapêuticas para minimizar esses danos funcionais.

\section{Contribuições dos autores}

Oliveira VCS participou do delineamento, busca, análise estatística dos dados da pesquisa, interpretação dos resultados e redação do artigo científico. Santos AP participou do delineamento, busca, interpretação dos resultados e redação do artigo científico. Castro IPR participou da concepção, coleta de dados da pesquisa, interpretação dos dados, delineamento e redação do artigo científico. Anjos JLM participou da concepção, coleta de dados da pesquisa e interpretação dos dados.

\section{Conflitos de interesses}

Nenhum conflito financeiro, legal ou político envolvendo terceiros (governo, empresas e fundações privadas, etc.) foi declarado para nenhum aspecto do trabalho submetido (incluindo, mas não se limitando a subvenções e financiamentos, participação em conselho consultivo, desenho de estudo, preparação de manuscrito, análise estatística, etc.).

\section{Referências}

1. Vlak, MH, Algra A, Brandenburg R, Rinkel GJ. Prevalence of unruptured intracranial aneurysms, with emphasis on sex, age, comorbidity, country, and time period: a systematic review and meta-analysis. Lancet Neurol 2011;10(7):626-36. doi: 10.1016/ S1474-4422(11)70109-0

2. Ministério da Saúde. Secretaria de Atenção à Saúde - SAS. Linha de cuidados em acidente vascular cerebral (AVC) na rede de atenção às urgências e emergências [Internet]. 2012. Disponível em: http://bvsms.saude.gov.br/bvs/publicacoes/manual_rotinas_ para_atencao_avc.pdf

3. Silva GS, Miranda RCAN, Massaud RM. Acidente Vascular Cerebral -Prevenção, Tratamento Agudo e Reabilitação. São Paulo: Atheneu; 2015.

4. Zhang J, Claterbuck RE. Molecular genetics of human intracranial aneurysms. Int J Stroke. 2008;3(4):272-87. doi: 10.1111/j.1747-4949.2008.00224.x

5. Woo D, Khoury J, Haverbusch MM, Sekar P, Flaherty ML, Kleidorfer DO et al.. Smoking and family history and risk of aneurysmal subarachnoid hemorrhage. Neurology. 2009;72(1);6972. doi: $10.1212 / 01$.wnl.0000338567.90260.46

6. Tiensoli SD, Bonisson RL, Matozinhos FP, Meléndez GV, Velásquez FSL. Diagnóstico situacional: perfil sociodemográfico e clínico de pacientes internados em unidade de clínica médica. Rev Min Enferm. 2014;18(3):573-578. doi: 10.5935/14152762.20140042 
7. Marques JC, Silva FAR, Martins AN, Perdigão FSO, Prudente COM, Fagundes RR. Profile of patients with sequelae of stroke in a rehabilitation center. Acta Fisiatr. 2019;26(3):144-148. doi: 10.11606/issn.2317-0190.v26i3a168160

8. Teasdale G, Jennett, B. Assessment of coma and impaired consciousness. Lancet. 1974;2(7872):81-4. doi: 10.1016/s01406736(74)91639-0

9. Gregson JM, Leathley MJ, Moore AP, Smith TL, Sharma AK Watkins CL. Reliability of measurements of muscle tone and muscle power in stroke patients. Age Ageing. 2000;29(3):223-8. doi: $10.1093 /$ ageing/29.3.223

10. Haan R, Limburg M, Bossuyt P, Meulen JVD, Aaronson N. The clinical meaning of rankin handicap grades after stroke. Stroke. 1995;26(11):2027-30. doi: 10.1161/01.str.26.11.2027

11. Fujiwara T, Liu M, Tsuji T, Sonoda S, Mizuno K, Akaboshi K et al. Development of a new measure to assess trunk impairment after stroke (trunk impairment scale): its psychometric properties. Am J Phys Med Rehabil. 2004;83(4):681-688. doi: 10.1097/01. PHM.0000137308.10562.20

12. Isaías LCS, Shimizu IS, Lavôr TBSL, Cunha GSD. Evolução Clínica de Pacientes com Aneurisma Cerebral Internados em um Hospital Público. RIES. 2018;7(2):156-167. doi: 10.33362/ries. $\underline{\mathrm{v} 7 \mathrm{i} 2.1436}$

13. Von Steinkirch C, Kato MVF, Chyla MM, Correia VB, Leal AG. Avaliação dos Aneurismas Intracranianos Tratados no Instituto de Neurologia de Curitiba. J Bras Neurocirurg. 2017;28(3):159-166. doi: $10.22290 / j b n c . v 28 i 3.1661$
14. Faleiro LCM, Pimenta NJG, Faleiro RM, Costa RA, Esmeraldo AC. Tratamento cirúrgico dos aneurismas não rotos da artéria cerebral média. Arq Neuropsiquiatr. 2004;62(2):319-321. doi: 10.1590/S0004-282X2004000200023

15. Cardozo Junior LCM, Barros BP, Holanda MFC. Fatores de risco em pacientes com aneurismas intracraniano atendidos em um hospital de referência de Belém - PA. J Bras Neurocirurg. 2011;22(3):95-99. doi: 10.22290/jbnc.v22i3.1011

16. Mocco J, Komotar RJ, Lavine SD, Meyers PM, Connolly ES, Solomon RA. The natural history of unruptured intracranial aneurysms. Neurosurg Focus. 2004;17(5):E3. doi: 10.3171/ foc.2004.17.5.3

17. Guaresi, JR, lung TC, Branco LTO, Medeiros MS, Sakae TM. Sequelas em pacientes com hemorragia subaracnóidea por ruptura de aneurisma intracraniano. ACM Arq Aatarin Med. 2011;40(2):34-40.

18. Loureiro AB, Vivas MC, Cacho RO, Cacho EWA, Borges G. Evolução Funcional de Pacientes com Hemorragia Subaracnóide Aneurismática não Traumática. R Bras Ci Saúde. 2015;19(2):123128. doi: $10.4034 /$ RBCS.2015.19.02.06 